Journal of Animal and Veterinary Advances 11 (7): 1041-1045, 2012

ISSN: $1680-5593$

(C) Medwell Journals, 2012

\title{
Product Fat-1 Transgenic Simmental Crossbred Cattle Endogenously Synthesizing Omega-3 Polyunsaturated Fatty Acids Using OSM
}

\author{
Wei Wang, Xiao-Mao Guo, Jian Wang and Song-Jia Lai \\ College of Animal Science and Technology, Sichuan Agricultural University, \\ Xinkang Road, 625014 Ya'an, Sichuan, China
}

\begin{abstract}
Meat products normally contain low omega-3 fatty acids and high ratio of n- $6 / \mathrm{n}-3$ fatty acids may contribute to the prevalence of many diseases. In order to obtained in such beef with high levels of omega-3 fatty acids will be more healthy to meet people's diet, researchers generated the fat- 1 transgenic simmental crossbred cattle expressing the Caenorhabditis elegans c fat-1 gene encoding an omega- 3 fatty acid desaturase that converts omega- 6 to omega- 3 fatty acids and the gene is absent in mammals. In this study, researchers successful product the embryo of fat-1 transgenic simmental crossbred cattle using OSM. The results of Western Blotting and fluoroscopic examination have proved that the fat-1 gene has been into genome of clone embryo and translated into proteins which could be used to embryo transfer.
\end{abstract}

Key words: OSM, fat-1, omega-3 fatty acid desaturase, omega-3 fatty acids, embryo, China

\section{INTRODUCTION}

Polyunsaturated Fatty Acids (PUFAs) are essential structural components of body. Omega-3 Polyunsaturated Fatty Acids (Omega-3 PUFA) as one importent type of PUFAs are crucial for life (Li-Hong and Xiao-Mei, 2006). The omega-3 PUFAs are important structural components of neuronal membranes and are involved in modulating neurotransmission, cell signaling, gene expression (Rapport, 2003; Kitajka et al., 2004) and enzyme activity (Murphy, 1990). Present studies in humans and animals have shown that omega-3 PUFAs have therapeutic use in patients who suffer from arrhythmia (Kang and Leaf, 1996) atherosclerosis (Kris-Etherton et al., 2002), hypertension (Howe, 1997) and hyperlipemia (Grimsgaard et al., 1997). Docosahexaenoic acid (22:6n-3; DHA) as one of the most abundant omega-3 Polyunsaturated Fatty Acid (PUFA) is required for normal neuronal development and function (Alessandri et al., 2004). Which is a potential factor for treat epilepsy (Schlanger et al., 2002; Yuen et al., 2005) and neurological disorder characterized by spontaneous and recurrent seizures (Burnham, 2006). Recently studies have suggest that omega-3 PUFAs have a major role to play in inhibiting the growth and evolution of multiple different systemic malignancies. For instance, omega-3 PUFAs could decrease the risk for colorectal cancer (Hall et al., 2008) prevent the evolution and development of prostate carcinomas (Mina et al., 2008) inducing apoptosis in chemoresistant pancreatic carcinomatous tissue (Hering et al., 2007), demonstrate antiproliferative effects in breast carcinomas (Sun et al., 2008) and gastric carcinoma (Otto et al., 2008).

The omega-3 PUFAs cannot be produced in the body and hence must be supplied in the food. Meat products normally contain small amounts of omega- 3 fatty acids and large amounts of omega- 6 fatty acids (Simopoulos, 1998; Hlophe and Moyo, 2011). Diets with a high ratio of omega-6/omega-3 fatty acids may contribute to the prevalence of many diseases such as coronary artery disease, cancer, diabetes, arthritis and depression (Simopoulos and Cleland, 2003). However, some lower life such as the roundworm Caenorhabditis elegans $c$ harbors a gene called fat-1 (Spychalla et al., 1997) which encodes an omega-3 fatty acid desaturase that can introduce a double bond into omega- 6 fatty acids at the omega-3 position of their hydrocarbon chains to form omega-3 fatty acids (Kang et al., 2001). Therefore, it is imperative to develop a transgenic cattle that carry fat-1 gene from Caenorhabditis elegans $c$ so that obtained in such beef with high levels of omega- 3 fatty acids will be more healthy to meet people's diet. Earlier research in transgenic mice has suggested the feasibility of creating fat-1 transgenic livestock capable of producing omega-3 fatty acids from the corresponding omega- 6 fatty acids.

In this study, One-Step Micromanipulation (OSM) has been used to generate the transgenic cattle. Which

Corresponding Author: Song-Jia Lai, College of Animal Science and Technology, Sichuan Agriculture University, 46\# Xinkang Road, 625014 Ya'an, Sichuan Province, China 
one is a modified approach to SCNT successfully used in the rat (Campbell, 2003) along with limited progress in the monkey (Mitalipov et al., 2002; Simerly and Navara, 2003) another species that has been difficult to clone. The process of SCNT involves placing a donor cell in the perivitelline space of a cytoplast and fusing donor and recipient cells with electrical pulses whereas in the OSM, a donor nucleus is isolated and directly injected into the cytoplast. Two protocols that are currently used to produce viable SCNT embryos and OSM was found to be significantly better than the Electrofusion Method. In the study, researchers collected oocytes and ear skin fibroblast cells of simmental crossbred cattle and produce simmental crossbred cattle's viable embryos capable of converting n-6 to n-3 fatty acids with the fat- 1 gene.

\section{MATERIALS AND METHODS}

Collection and culture of cumulus-oocyte complexes: Ovaries were collected from prepubertal female simmental crossbred cattle at a local abattoir and transported to the laboratory in $0.9 \% \mathrm{NaCl}$ solution at $35-39^{\circ} \mathrm{C}$. CumulusOocyte Complexes (COCs) were aspirated from antral follicles (2-6 $\mathrm{mm}$ in diameter) with an 20-gauge disposable syringe. COCs were washed three times in PBS buffer containing 10\% Fetal Bovine Serum (FBS) and 50-60 COCs were transferred to $500 \mathrm{~mL}$ of the TCM199 maturation

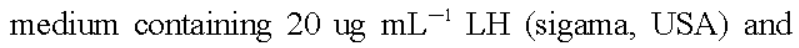
10 ug $\mathrm{mL}^{-1} \mathrm{FSH}$ (sigama, USA) that had been covered with mineral oil in a 4-well multidish and equilibrated at $39^{\circ} \mathrm{C}$ in an atmosphere of $5 \% \mathrm{CO}_{2}$ in air overnight. The oocyte will be matured until being at collection (MII), before SCNT.

Donor cell culture: A small ear skin biopsy was obtained from prepubertal female simmental crossbred cattle at a local abattoir and the tissue was cut into small pieces with fine scissors. The cells were incubated at $37^{\circ} \mathrm{C}$ in PBS containing $0.05 \%$ trypsin and $0.5 \mathrm{mM}$ EDTA overnight and this suspension was centrifuged. The cell pellet was resuspended and cultured in DMEM-F12 medium supplemented with $75 \mathrm{mg} \mathrm{mL}^{-1}$ penicillin $\mathrm{G}, 50 \mathrm{mg} \mathrm{mL}^{-1}$ streptomycin and 15\% (v:v) Fetal Calf Serum (FCS).

Plasmid construction and transfection: The fat-1 expression vector, PEGFP-N1 -fat-1 which contains a fat-1 cDNA and one eGFP sequence driven by the CMV promoter. The fat-1 gene was clone from Caenorhabditis elegans c (contributed by Department of Parasitology, China Agricultural University). Primers for fat- 1 gene were 5'-ATGGTCGCTCATTCCTCAG-3' (forw-ard) and 5'AGTTATGGCTTTATGCATTCAA-3' (reverse), 5'- CCG
GAATTC ATGGTCGCTCATTCCTCAG-3' (forward) and 5'-CGCGGATCC AGTTATGGCTTTATGCATTCAA-3' (reverse). The fibroblasts were transfected by X-fectmediated (TAKARA, Japen) plasmid PEGFP-N1-fat-1. After G418 selection, surviving cells were used as a nuclear donor.

Spindle removal, nuclear transfer and activation: The OSM technique described previously was employed. Briefly, a fibroblast was aspirated into a $10 \mu \mathrm{m}$ ID blunt-tip pipette when the cell membrane lysed. The cell was then injected into an oocyte at the side opposite the MII spindle after the injection pipette was passed through the zona and oolemma and then the injection pipette was slowly withdrawn into proximity to the metaphase chromosomes as visualized by micromanipulator. The chromosomes along with a small amount of ooplasm were aspirated into the pipette removed from the oocyte and stained with Hoechst 33342 in a separate drop to confirm spindle removal. About $2 \mathrm{~h}$ later, activation was induced by exposure to $5 \mu \mathrm{M}$ ionomycin for $4 \mathrm{~min}$ and then exposure to $2 \mathrm{mM}$ 6-Dimethylaminopurine (DMAP) for $5 \mathrm{~h}$ at $37^{\circ} \mathrm{C}$ in $5 \% \mathrm{CO}_{2}$-balance air. Activated SCNT embryos were cultured in SOFaa culture solution containing $10 \% \mathrm{FCS}$ at $37^{\circ} \mathrm{C}$ in $5 \% \mathrm{CO}_{2}$-balance air.

Western blotting: The concentration of proteins was measured by Bradford reagent (Sigma), eparated on 10\% SDS-PAGE gels and transferred to Immobilon membranes (Millipore). After blocking in 5\% low-fat milk in BST $(0.1 \%$ Tween 20 in PBS) for $1 \mathrm{~h}$, the membranes were ncubated with GFP Ab-2 antibody (1:500, sigama, USA) horseradish peroxidase labeled sheep anti-rat (1:2000, sigama, USA) overnight at $4^{\circ} \mathrm{C}$ After washing in PBST, the membranes were incubated in goat anti-rabbit antibody conjuated with horseradish peroxidase $(1: 5000)$ for $1 \mathrm{~h}$ followed by hree washes in PBST. The signals were detected by Gel imaging system.

Fluoroscopic examination: The fat- 1 transfected fibroblast and activated embryos were observed by fluorescence microscope (Nikon, Japen) using objective and excitation wavelengths of $\mathrm{CY} 3$ and FITC in order to select the ones carried $e G F P$ and fat-1 gene. For each experiment, the same detector gain, amplifier offset and pinhole parameters were used.

\section{RESULTS AND DISCUSSION}

The previous studies have shown that the fat-1 mice have the capable of de novo synthesis of n-3 PUFA from $n-6$ PUFA. In these studies, the transgenic fat-1 


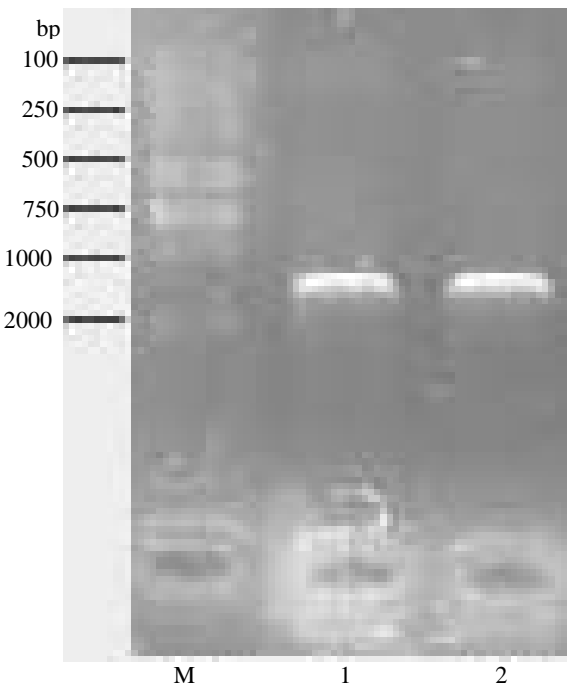

Fig. 1: Polymerase chain reaction amplifications of $C$. elegans fat-1; M: Molecular size markers DL2000 and 1,2: Amplifications of C. elegans fat-1

mouse was as a model to study the pathophysiology of cardiovascular, neurological and psychiatric disorders (Das and Puskas, 2009) to prevent neoplasia (Griffitts et al., 2010), to reduction of inflammation and chronic tissue damage (Weylandt et al., 2008) and to improve glucose tolerance (Smith et al., 2010). Rarely, study aim at providing meat products which are high in low in omega-3 fatty acids. In this study, we successful product the embryo of fat-1 transgenic simmental crossbred cattle using OSM. The complete nucleotide sequence of Caenorhabditis elegans $\mathrm{c}$ fat-1 cDNA of was amplified (Fig. 1) and which was corrected according to GeneBank (Accession No. NM_001028389). This cDNA sequence was a single open reading frame without any signal peptide and which encodes a putative polypeptide of 454 amino acids (aa) and the molecular mass of polypeptide was $52.912 \mathrm{kDa}$ according to the result of Signal PV3.0 Server analysis.

On the basis of correct sequence result of fat-1 cDNA, researchers inset fat- $1 \mathrm{cDNA}$ at the sites of EcoRI and BamHI to construct the eukaryotic expression vector. The recombinant plasmid with the fat-1 gene was correct by digestion identification (Fig. 2). To determine the expression of fat-1 proteins in fibroblasts, the eGFP was connected to the fat- 1 expression vector. Through determine the signal of eGFP and fat-1 proteins can be distinguished in fibroblasts. The fat-1 transfected fibroblasts were subsequently cultured in $250 \mathrm{ug} \mathrm{mL} \mathrm{m}^{-1}$ G418, the survivals were almost all have fluorescence

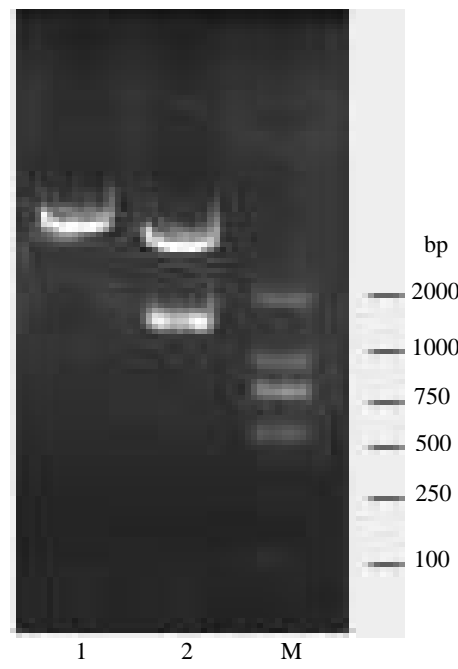

Fig. 2: Restriction map of recombinant plasmid; M: DNA Marker D2000; 1: Double-enzyme digested products and 2: Plasmid control

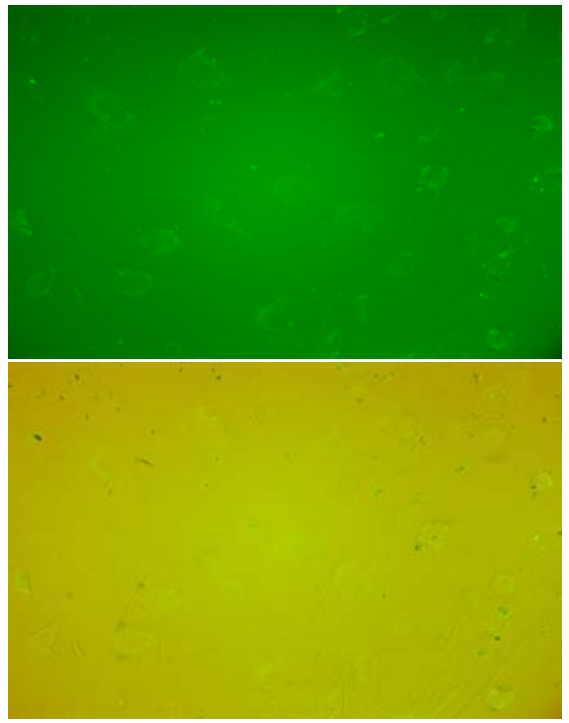

Fig. 3: Transgenic cell clones after selection for 7 days with G418; bright-field pictures of cells on the right; corresponding images under a fluorescence microscope on the left (Wavelengths: CY3 and FITC; magnification: 10x40)

signal. As shown in the (Fig. 3), the signals were detected in cytoplasm and cytomembrane but not in nucleus. What is the same as the previous description (Murphy, 1990). The survivals carried signals were select and been used to Western blotting. By Western blotting, the protein of fat-1 was evaluated by immunoblot analysis using recombinant protein which was $72.9 \mathrm{kDa}$ consisted of a $52.9 \mathrm{kDa}$ of fat-1 gene protein and $27 \mathrm{kDa}$ of eGFP 


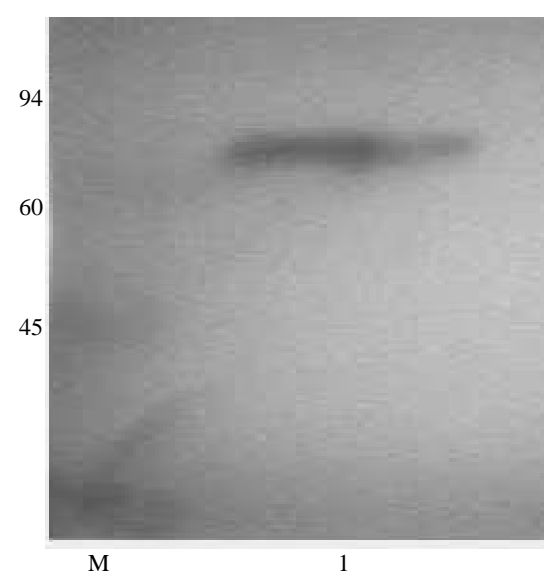

Fig. 4: Western-blotting of expressed recombinant protein; $\mathrm{M}$ : Protein marker (Unit: $\mathrm{kDa}$ ) and 1: Western-blotting of expressed recombinant proteins

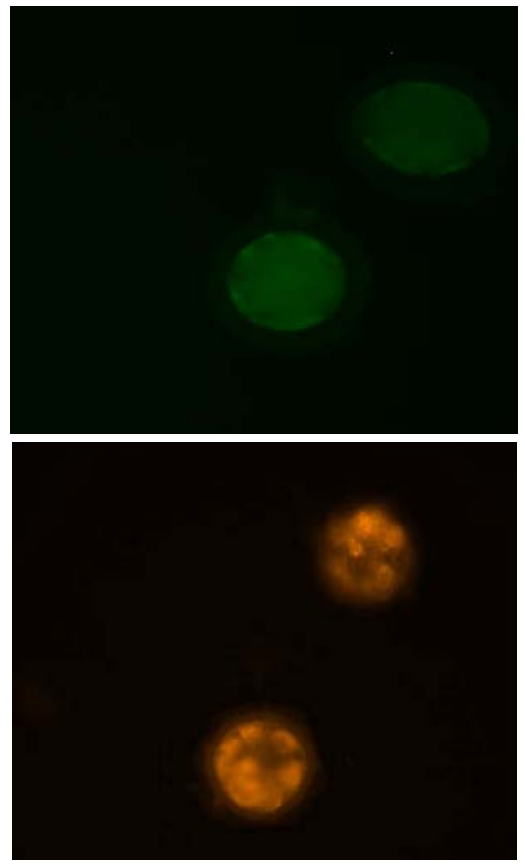

Fig. 5: GFP-positive embryos expressing the transgene from pGFP-N1-fatl plasmid; the 8-cell embryos under the fluorescence microscope using the wavelengths of RED on the left; corresponding the morulaes using the wavelengths of FICT on the right (magnification: $10 \times 10$ )

protein (Fig. 4). Researchers could certain this protein was correct corroding to the electrophoretogram. Researchers could be sure that the fat 1 gene have been into genome of fibroblasts and translated into proteins according to these evidences. The survivals could be used to OSM as donor. In the study, the early embryos which carried the fat-1 gene have also been examined by fluorescence microscope. Researchers examined three preimplantation stages include two-cells, four-cells and blastocyst. After cleavage, fluorescence signals were characterized by the presence of larger and even more intensely foci in the blastomere of embryos (Fig. 5).

\section{CONCLUSION}

The study shows that strong signal were found everywhere in the blastocyst especially in the periphery of blastocyst but there were not any fluorescence signals in transparent zone. So, the blastocyst which carried the fat 1 gene could be used to embryo transfer and then the generation of cloned cattle will be examining by gas chromatography in order to the level of omega- 3 fatty acids from omega- 6 analogs in future study. In addition, the fat-1 transgenic simmental crossbred cattles provide a large animal model in which to product such beef with high levels of omega-3 fatty acids will be more healthy to meet people's diet.

\section{ACKNOWLEDGEMENT}

This study was partially supported by the Research of Breeding New Transgenic Organisms (2009ZX08007005B-09).

\section{REFERENCES}

Alessandri, J.M., P. Guesnet, S. Vancassel, P. Astorg and I. Denis et al., 2004. Polyunsaturated fatty acids in the central nervous system: Evolution of concepts and nutritional implications throughout life. Reprod. Nutr. Dev., 44: 509-538.

Burnham, W.M., 2006. Principles of Medical Pharmacology. 7th Edn., Elsevier, Amsterdam. Pages: 223.

Campbell, N., 2003. I smell a rat. Nat. Rev. Genet., 4: 853-853.

Das, U.N. and L.G. Puskas, 2009. Transgenic fat-1 mouse as a model to study the pathophysiology of cardiovascular, neurological and psychiatric disorders. Lipids Health Dis., 10.1186/1476-511X-8-61.

Griffitts, J., D. Saunders, Y.A. Tesiram, G.E. Reid and A. Salih et al., 2010. Non-mammalian fat-1 gene prevents neoplasia when introduced to a mouse hepatocarcinogenesis model: Omega-3 fatty acids prevent liver neoplasia. Biochim. Biophys. Acta, 10: 1133-1144. 
Grimsgaard, S., K.H. Bonaa, J.B. Hansen and A. Nordoy, 1997. Highly purified eicosapentaenoic acid and docosahexenoic acid in humans have similar triacylglycerol-lowering effects but divergent effects on gerum fatty acids. Am. J. Clin. Nutr., 66: 649-659.

Hall, M.N., J.E. Chavarro, I.M. Lee, W.C. Willett and J. Ma, 2008. A 22-year prospective study of fish, n-3 fatty acid intake and colorectal cancer risk in men. Cancer Epidemiol. Biomarkers Prev., 17: 1136-1143.

Hering, J., S. Garrean, T.R. Dekoj, A. Razzak and A. Saied et al., 2007. Inhibition of proliferation by omega-3 fatty acids in chemoresistant pancreatic cancer cells. Ann. Surg. Oncol., 14: 3620-3628.

Hlophe, S.N. and N.A.G. Moyo, 2011. The effect of different plant diets on the growth performance, gastric evacuation rate and carcass composition of Tilapia rendalli. Asian J. Anim. Vet. Adv., 6: 1001-1009.

Howe, P.R., 1997. Dietary fats and hypertension focus on fish oil. Ann. New York Acad. Sci., 827: 339-352.

Kang, J.X. and A. Leaf, 1996. Antiarrhythmic effects of polyunsaturated fatty acids: Recent studies. Circulation, 94: 1774-1780.

Kang., Z.B., Y. Ge, Z.H. Chen, J. Brown, M. Laposata, A. Leaf and J.X. Kang, 2001. Adenoviral gene transfer of C. elegans n-3 fatty acid desaturase optimizes fatty acid composition in mammalian cells. Proc. Natl. Acad. Sci., 98: 4050-4054.

Kitajka, K., A. Sinclair, R.S. Weisinger, H.S. Weisinger and M. Mathai et al., 2004. Effects of dietary omega-3 polyunsaturated fatty acids on brain gene expression. Proc. Natl. Acad. Sci., 101: 10931-10936.

Kris-Etherton, P.M., W.S. Harris and L.J. Appel, 2002. Fish consumption, fish oil, omega-3 fatty acids and cardiovascular disease. Circulation, 106: 2747-2757.

Li-Hong, M. and W. Xiao-Mei, 2006. Pharmacologic actions of polyunsaturated fatty acid. J. Jilin Sci. Chinese Trad. Med., 6: 69-70.

Mina, K., L. Fritschi, K. Johnson and T.C. Group, 2008. An inverse association between preserved fish and prostate cancer: results from a population-based case-control study in Canada. Nutr. Cancer, 60: 222-226.

Mitalipov, S.M., R.R. Yeoman, K.D. Nusser andD.P. Wolf, 2002. Rhesus monkey embryos produced by nuclear transfer from embryonic blastomeres or somatic cells. Biol. Reprod., 66: 1367-1373.
Murphy, M.G., 1990. Dietary fatty acids and membrane protein function. J. Nutr. Biochem., 1: 68-79.

Otto, C., U. Kaemmerer, B. Illert, B. Muehling and N. Pfetzer et al., 2008. Growth of human gastric cancer cells in nude mice is delayed by a ketogenic diet supplemented with omega-3 fatty acids and medium-chain triglycerides. BMC Cancer, 8: 122-122.

Rapport, S.I., 2003. In vivo approaches to quantifying and imaging brain arachidonic and docosahexaenoic acid metabolism. J. Pediatr., 143: 26-34.

Schlanger, S., M. Shinitzky and D. Yam, 2002. Diet enriched in omega- 3 fatty acids alleviates convulsion symptoms in epilepsy patients. Epilepsia, 43: 103-104.

Simerly, C.R. and C.S. Navara, 2003. Nuclear transfer in the rhesus monkey: Opportunities and challenges. Clon. Stem Cells, 5: 319-331.

Simopoulos, A.P. andL.G. Cleland, 2003. Omega-6/omega3 essential fatty acids ratio: The scientific evidence. World Rev. Nutr. Diet., 92: 74-80.

Simopoulos, A.P., 1998. Evolutionary aspects of diet and essential fatty acids. World Rev. Nutr. Diet., 83: 1-11.

Smith, B.K., G.P. Holloway, S. Reza-Lopez, S.M. Jeram, J.X. Kang Kang and D.W. Ma, 2010. A decreased n$6 / n-3$ ratio in the fat- 1 mouse is associated with improved glucose tolerance. Applied Physiol. Nutr. Metab., 35: 699-706.

Spychalla, J.P., A.J. Kinney and J. Browse, 1997. Identi?cation of an animal omega-3 fatty acid desaturase by heterologous expression in Arabidopsis. Proc. Natl. Acad. Sci. USA., 94: 1142-1147.

Sun, H., I.M. Berquin, R.T. Owens, J.T. O'Flaherty and I.J. Edwards, 2008. Peroxisome proliferator-activated receptor $\gamma$-mediated up-regulation of syndecan- 1 by n-3 fatty acids promotes apoptosis of human breast cancer cells. Cancer Res., 68: 2912-2919.

Weylandt, K.H., A. Nadolny, L. Kahlke, T. Kohnke and C. Schmocker et al., 2008. Reduction of inflammation and chronic tissue damage by omega-3 fatty acids in fat-1 transgenic mice with pancreatitis. Biochim. Biophys. Acta, 1782: 634-641.

Yuen, A.W., J.W. Sander, D. Fluegel, P.N. Patsalos, G.S. Bell, T. Johnson and M.J. Koepp, 2005. Omega-3 fatty acid supplementation in patients with chronic epilepsy: A randomized trial. Epilepsy Behav., 7: 253-258. 